\title{
Editorial
}

\section{Complex Discrete Dynamics and Its Structures in Bioinspired Systems}

\author{
Gualberto Solís-Perales, ${ }^{1}$ Ricardo Femat, ${ }^{2}$ Kwok-Wo Wong, ${ }^{3}$ and Eric Campos-Cantón ${ }^{2}$ \\ ${ }^{1}$ Departamento de Electrónica, CUCEI, Universidad de Guadalajara, Avenida Revolución 1500, CP 44430, Guadalajara, JAL, Mexico \\ ${ }^{2}$ División de Matemáticas Aplicadas, Instituto Potosino de Investigación Científica y Tecnológica, Camino a la Presa San José 2055, \\ Colonia Lomas 4 Sección, CP 78216, San Luis Potosí, SLP, Mexico \\ ${ }^{3}$ Department of Electronic Engineering, City University of Hong Kong, Kowloon, Hong Kong
}

Correspondence should be addressed to Gualberto Solís-Perales; gualberto.solis@cucei.udg.mx

Received 1 April 2013; Accepted 1 April 2013

Copyright (C) 2013 Gualberto Solís-Perales et al. This is an open access article distributed under the Creative Commons Attribution License, which permits unrestricted use, distribution, and reproduction in any medium, provided the original work is properly cited.

Understanding the dynamical behavior of bioinspired systems that interact with so many variables or agents is an amazing challenge. Dealing with a single model provides understanding of the phenomena itself; however, studying interactions between many systems provides relevant information about the collective dynamic behavior and it is one of the most interesting and recent paradigms in nonlinear science. This kind of complex systems is hard to handle in a completely deterministic manner, so the goal seems to be reached when it is possible to reproduce a similar behavior by means of mathematical models. Furthermore, these models provide us with information on how these bioinspired systems can be controlled and tell us about their collective dynamics. Thus, a characterization of the system behavior is useful and possible and today remains a challenge.

This special issue contains contribution in this direction. Among topics included, the reader can find wide themes from the multiagent robot to $\beta$ cells passing through mechatronics and biomedicine.

The paper entitled "Discrete pseudo-SINR-balancing nonlinear recurrent system" by Z. Uykan presents the concept of pseudo-signal-to-interference-noise-ratio (pseudo-SINR) which is employed in the stability analysis of a discretetime recurrent neural network. The results show that the instantaneous pseudo-SINRs are balanced when the network state approaches an equilibrium point. Some applications of the proposed network as an associative memory and in solving the clustering problem are given.
The paper entitled "Existence and global stability of a periodic solution for discrete-time cellular neural networks" by $\mathrm{H}$. Shao et al. presents the existence of periodic solution for a discrete-time cellular neural network (DT-CNN) in the three-dimensional space which is proven using the continuation theorem of coincidence degree theory. The global stability of the periodic solution is analyzed utilizing the Lyapunov stability theory. The results are verified by numerical simulation on an example of cellular neural network.

The paper "Decentralized discrete-time formation control for multi-robot systems" by E. G. Hernandez-Martinez et al. presents a discrete-time analysis of the formation control of mobile robots. The analysis is carried out in a group of mobile robots, where only the position of a certain number of them is available which means that the robot does not possess the position of all the robots. The main contribution consists in providing the global convergence to the formation pattern represented by an arbitrary formation graph using attractive potential functions.

The paper entitled "Discrete coupling and synchronization in the insulin release in the mathematical model of the $\beta$ cells" by L. J. Ontañón-García and E. Campos-Cantón presents the generation of bursting electrical activity in $\beta$ cells. The behaviors which are studied are the active, inactive, and continuous spiking using discrete couplings. The contribution considers two stages: one consists in a mechanism on how to switch between the behaviors and the second is based on how to deal with the in-phase synchronization between active cells. 
The paper entitled "Facial edema evaluation using digital image processing" by A. E. Villafuerte-Nuñez et al. presents a system that measures the four main variables present in facial edemas: trismus, blush (coloration), temperature, and inflammation. Measurements are obtained by using image processing and the combination of different devices such as a projector, a PC, a digital camera, a thermographic camera, and a cephalostat. Some tests using controlled measurements of every variable are presented in this paper. The results allow evaluating the measurement system before its use in a real test, which consists in extracting the third molar to generate the facial edema.

The paper entitled "Single Gaussian chaotic neuron: numerical study and implementation in an embedded system" by L. M. Torres-Treviño et al. presents the analysis of complex dynamic systems inspired by neurons. These complex systems are called artificial Gaussian neurons due to use of a Gaussian activation function that includes two parameters called the center of mass and sensibility factor. The behavior of the neuron is parametric characterized and there are values of the parameters where the neuron displays chaotic behavior.

The paper entitled "Towards human capture movement: estimation of anatomical movements of the shoulder" by B. B. Salmerón-Quiroz et al. presents the human arm motion for human root integration and for visualising the movement in a virtual model for robot control. The technique uses MEMS and information of the anatomy of the arm, from where the arm position, the anatomical movements and the accelerations of the shoulder can be estimated. This with the aim of physical rehabilitation and training of stroke patients. The procedure uses a nonlinear observer and a discrete optimization routine to fuse the information from the sensors and to track a desired trajectory in the shoulder in the presence of uncertainties.

The paper entitled "Vulnerability analysis of power grids using modified centrality measures" by F. Gutierrez et al. presents a modification of centrality measures as a tool to identify critical nodes in an electrical power grid. The basis to define closeness and betweenness of the nodes is the grid active power flow and, therefore, identifying the most critical nodes. Finally, the information obtained via modified centrality measures is used to calculate global efficiency of the power grids.

The paper entitled "Analysis of a dengue disease model with nonlinear incidence" by S.-M. Guo et al. presents a model of a biosystem based on dengue disease epidemic. The model considers nonlinear incidence which produces rich dynamics and the stability of the system is analyzed through a geometric approach to stability.

The paper entitled "Generation of a reconfigurable logical cell using evolutionary computation” by I. Campos-Cantón et al. presents a strategy to reconfigure a logical cell, which is based on the tent map, which is a simple discrete system. The reconfiguration of the logical cell is carried out by means of variations in the parameters of the system in the cell, and in this way the cell presents several logical functions without changing the cell. The parameters are tuned via a chaotic system and an evolutionary algorithm is tuned via a trial error proses.
The paper entitled "A network model on credit risk contagion" by T.-Q. Chen and J.-M. He shows and describes a network model of credit risk contagion where the effect of behavior of credit risk holders, the financial market regulators, and the network structure are considered. The effect mechanisms of the degree of individual relationship, individual attitude to credit risk contagion, the individual ability to resist credit risk contagion, the monitoring strength of the financial market regulators, and the network structure on credit risk contagion are discussed using the stochastic dominance theory.

\section{Acknowledgment}

Guest Editors wish to thank authors contributing this discussion of bio-inspired dynamical studies.

Gualberto Solís-Perales Ricardo Femat Kwok-Wo Wong Eric Campos-Cantón 


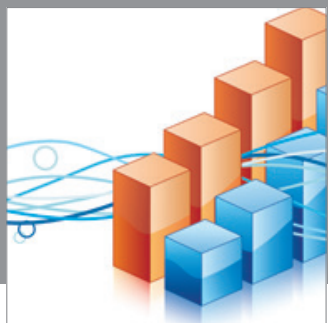

Advances in

Operations Research

mansans

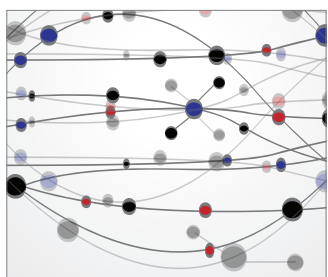

The Scientific World Journal
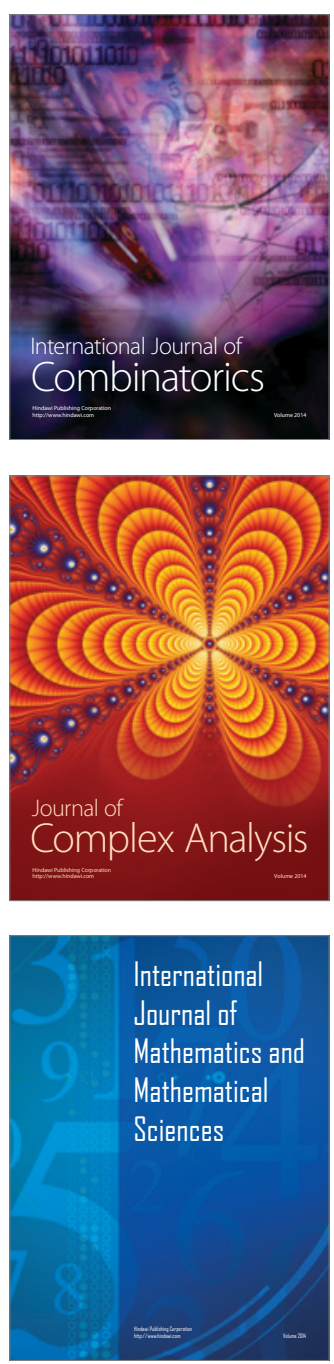
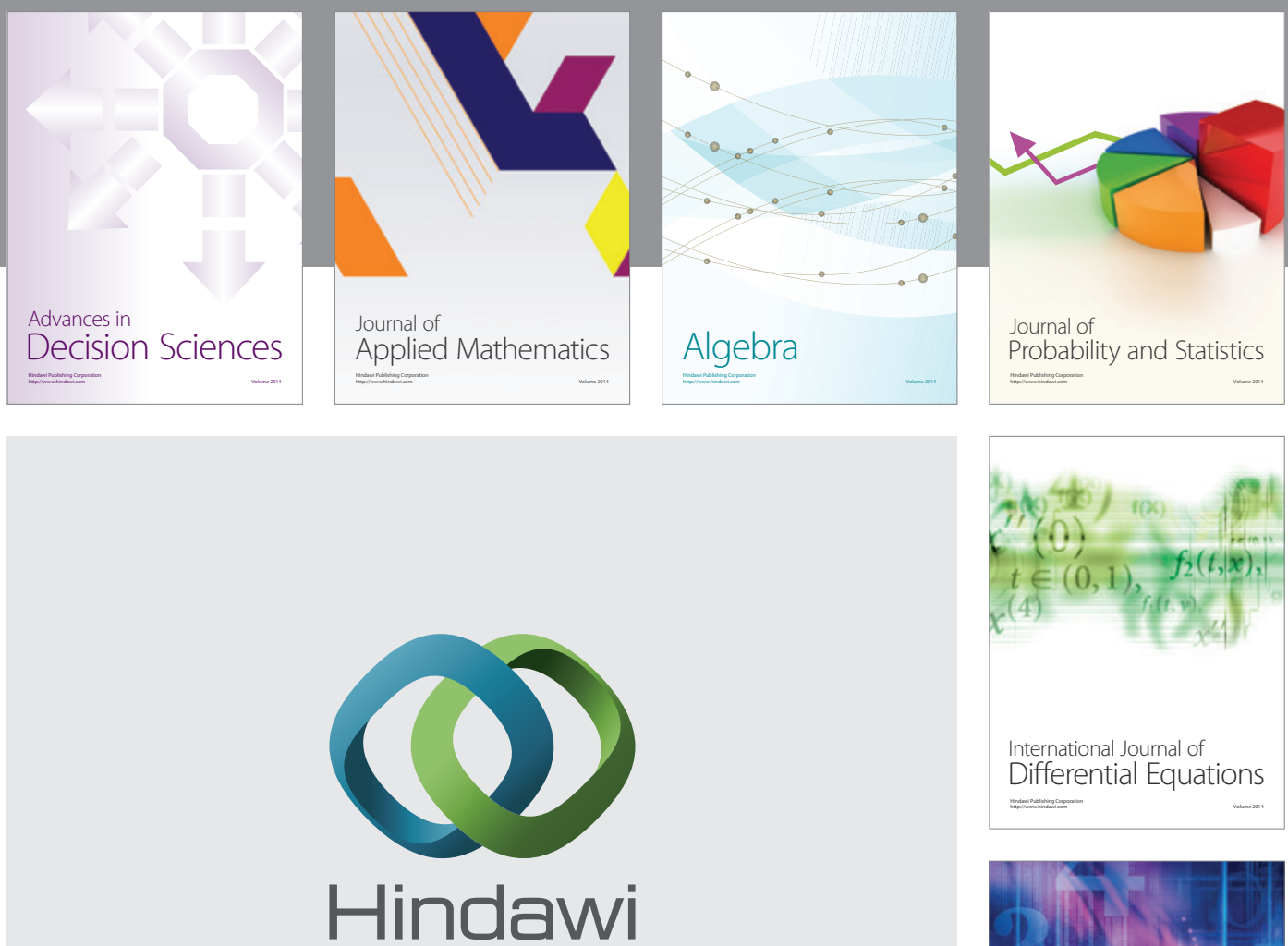

Submit your manuscripts at http://www.hindawi.com


Journal of

Function Spaces

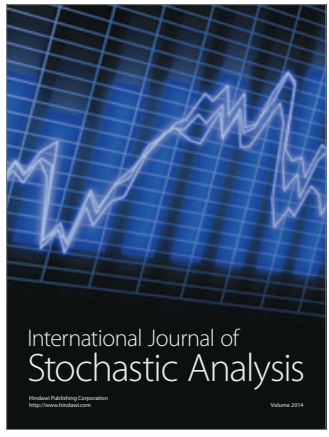

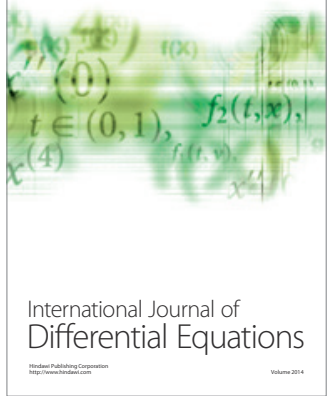
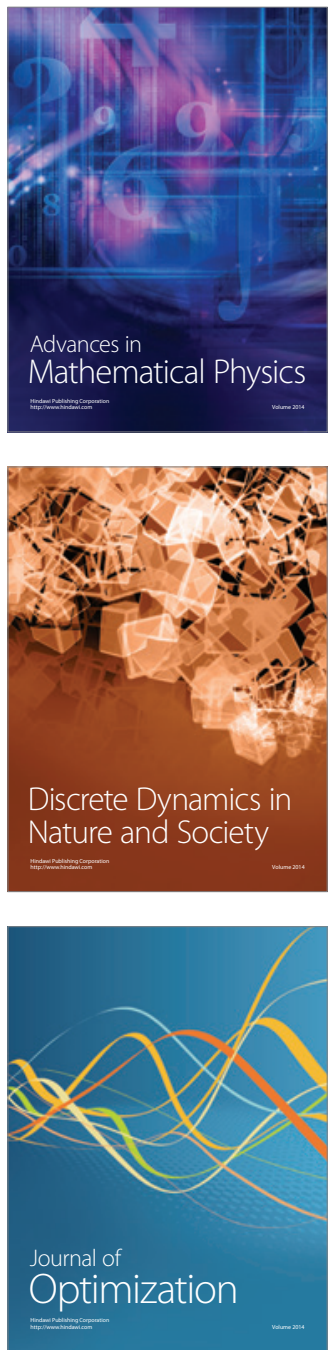